\title{
Lesiones renales y pancreáticas inducidas por Ipomoea carnea en cabras
}

\author{
Rios, E. '; Cholich, L. ${ }^{1}$; Teibler, G. ${ }^{1}$; Bogado, F. ${ }^{1}$; Mussart N. ${ }^{2}$ \\ ${ }^{1}$ Departamento Clínicas, ${ }^{2}$ Departamento Ciencias Básicas, Facultad de Ciencias Veterinarias, UNNE, \\ Sargento Cabral 2139, Corrientes (3400), Argentina, \\ Tel/Fax: 03783-425753, E-mail: patmed@vet.unne.edu.ar.
}

\begin{abstract}
Resumen
Rios, E.; Cholich, L.; Teibler, G.; Bogado, F.; Mussart, N.: Lesiones renales y pancreáticas inducidas por Ipomoea carnea en cabras. Rev. vet. 20: 1, 45-49, 2009. Ipomoea carnea es una planta tóxica debido a los alcaloides swainsonina y calisteginas, que provoca pérdidas económicas en la producción animal del nordeste argentino. El propósito de este estudio fue describir las lesiones microscópicas verificadas en páncreas y riñón, así como los cambios del perfil bioquímico renal causados por la intoxicación experimental con I. carnea en caprinos. Se emplearon 9 cabras alimentadas con alfalfa; 4 de ellas obraron como testigos y las 5 restantes fueron sometidas a la ingestión de hojas de $I$. carnea a razón de $50 \mathrm{~g} / \mathrm{kg}$ /día durante 53 días, fecha en que fueron sacrificadas debido al grave deterioro de su salud. Semanalmente se realizaron exámenes clínicos y extracciones de sangre y orina. Urea y creatinina incrementaron progresivamente su concentración sérica en los animales intoxicados, quienes también revelaron anormalidades en el urianálisis. Los órganos más afectados fueron páncreas y riñón. Además de la vacuolización citoplasmática descripta por otros autores, en el presente estudio se constató la presencia de contenido eosinofilico intratubular y ensanchamiento del espacio de Bowman en los riñones, daños que explicarían las anormalidades detectadas en orina y sangre. Tales cambios asumen utilidad al momento de realizar el diagnóstico de esta intoxicación.
\end{abstract}

Palabras clave: cabra, Ipomoea carnea, intoxicación, riñón, páncreas.

\begin{abstract}
Rios, E.; Cholich, L.; Teibler, G.; Bogado, F.; Mussart, N.: Renal and pancreatic damage induced by Ipomoea carnea in goats. Rev. vet. 20: 1, 45-49, 2009. Ipomoea carnea is a toxic plant due to the alkaloids swainsonine and calystegines, which causes animal production economic losses in Northeast Argentina. The purpose of this study was to describe microscopic lesions verified in pancreas and kidney, as well as renal biochemical profile changes caused by I. carnea experimental intoxication in goats. Nine goats fed on alfalfa hay were used; four of them were controls and the five remainder were submitted to the ingestion of $I$. carnea leaves $(50 \mathrm{~g} / \mathrm{kg} / \mathrm{day}$ during 53 days). At this time these animals were sacrificed due to its serious health deterioration. Clinical exams, as well as blood and urine sampling, were weekly carried out. Urea and creatinine progressively increased their serum concentration in the intoxicated animals, which also revealed abnormalities in the urine composition. Pancreas and kidneys were gravely affected. Besides the intracellular vacuolization already described by other authors, the presence of an intratubular eosinophillic content, as well as an expansion of Bowman space in the kidneys, were verified in present study. These damages would explain the abnormalities detected in urine and blood. Such changes are useful to optimize the diagnosis of this intoxication.
\end{abstract}

Key words: goat, Ipomoea carnea, intoxication, kidney, pancreas.

\section{INTRODUCCIÓN}

Ipomoea carnea var. fistulosa (Convolvulaceae) es una planta propia de zonas parcialmente inundables, cuya ingestión provoca intoxicación natural en animales de

Recibido: 6 mayo 2009 / Aceptado: 23 julio 2009 producción ${ }^{7}$. Ha sido reportada en regiones tropicales y subtropicales como Venezuela ${ }^{4}$, Mozambique ${ }^{5}$, Brasil $^{8}$ y Argentina ${ }^{16}$. Afecta principalmente a caprinos, ovinos y bovinos. El consumo de la planta se produce principalmente en épocas de escasez de pasturas naturales, como en la estación invernal, debido a que es una de las pocas especies que permanece verde todo el año ${ }^{15}$. 
Algunos animales muestran gran apetencia hacia el consumo de I. carnea, lo cual posiblemente conduzca a una adicción ${ }^{2,14}$. La signología clínica de la intoxicación se caracteriza por síntomas nerviosos como anormalidades en la marcha, estación y postura, emaciación, ataxia y posterior paresia 1,7,17,19. Otros signos observados son pérdida de peso, disminución de la frecuencia de ruidos ruminales, anemia, retardo en el tiempo de llenado capilar y anormalidad del pliegue cutáneo ${ }^{4,16}$. Los síntomas varían en frecuencia, progresión y severidad de la intoxicación ${ }^{1}$.

La toxicidad de I. carnea es atribuida a la presencia de alcaloides denominados swainsonina y calisteginas $\mathrm{B}_{1}, \mathrm{~B}_{2}$ y $\mathrm{C}_{1}$ presentes en hojas, semillas y flores 3, 7-9. Mientras que calistegina revela una potente actividad inhibitoria $\alpha$ y $\beta$-galactosidasa, así como de $\alpha$-glucosidasa, swainsonina es conocida como un potente inhibidor de la $\alpha$-manosidasa lisosomal $1,6,7,9,12$, 13, 18 . Estos alcaloides inducen una incompleta metabolización lisosomal de oligosacáridos, los cuales se acumulan en dichos organoides provocando anormalidades celulares funcionales y finalmente la muerte celular, a través de un proceso conocido como "enfermedad de almacenamiento lisosomal" 5,11 .

El propósito de este trabajo fue describir los cambios del perfil bioquímico renal y las lesiones histopatológicas de riñón y páncreas causadas por la intoxicación experimental de cabras por ingestión de I. carnea obtenida en áreas rurales del nordeste argentino, habida cuenta que este tipo de estudio aún no fue realizado en la región.

\section{MATERIAL Y MÉTODOS}

Muestras de I. carnea (tallos y hojas) fueron colectadas en temporada otoñal en predios del Departamento Capital de la Provincia de Corrientes, Argentina, e identificadas taxonómicamente por el Instituto de Botánica de la Facultad de Ciencias Agrarias de la UNNE.

Nueve caprinos de raza criolla, de ambos sexos, de 3 años de edad y clínicamente sanos, se dividieron en lote control $(\mathrm{n}=4$, alimentado con alfalfa) y lote experimental ( $\mathrm{n}=5$, alfalfa + hojas y tallos de $I$. carnea vía oral a razón de $50 \mathrm{~g} / \mathrm{kg} / \mathrm{día}$ ). Ambos grupos tuvieron acceso ad-libitum al agua. Semanalmente se efectuó examen clínico de los animales y extracciones de sangre y orina. La administración de la planta tóxica se extendió hasta los 53 días, período en el cual los animales mostraron síntomas intensos de intoxicación. Para tal fecha, cada ejemplar había consumido alrededor de $50-52 \mathrm{~kg}$ de planta.

Las pruebas de laboratorio consistieron en determinaciones séricas de urea (ureasa, $546 \mathrm{~nm}$ ) y creatinina (picrato alcalino, $510 \mathrm{~nm}$ ), efectuadas en un espectrofotómetro Zeltec ZL-500, utilizando reactivos Wiener Lab. El urianálisis incluyó la investigación del pH, densidad y presencia de nitritos, leucocitos, proteínas, glucosa, cetonas, urobilinógeno, bilirrubina y sangre (tiras reactivas UrineStrip, Wiener Lab). El examen microscópico del sedimento urinario comprendió la estimación de la cantidad y tipo de células, así como la presencia de cristales, cilindros y otras anormalidades.

Los animales fueron sacrificados acorde a la gravedad de los síntomas clínicos que presentaban, dado que algunos ya no podían mantenerse en estación y no podían alimentarse ni beber agua por sí solos. Fueron anestesiados con una combinación de clorhidrato de xilacina al $2 \%$ y clorhidrato de ketamina, para luego ser sometidos a una sangría "a blanco" a través de las arterias carótidas comunes, momento en que se procedió a extraer secciones de tejidos para análisis histopatológicos.

Muestras de páncreas y riñón fueron fijadas en formol al $10 \%$, obteniéndose secciones de $5 \mu \mathrm{m}$ que fueron coloreadas con hematoxilina-eosina (HyE) para su observación mediante microscopía óptica. En los cortes histológicos de corteza renal se procedió a medir el diámetro de los glomérulos y de los corpúsculos de Malpighi de cabras controles e intoxicadas, a fines de estimar -por diferencia entre ambos- la amplitud del espacio de Bowman.

Los valores numéricos fueron procesados estadísticamente para obtener medidas de tendencia central y dispersión. También se realizó análisis de variancia a una vía, utilizando un valor de significación del 5\%.

\section{RESULTADOS}

La concentración sérica de urea fue de $0,21 \pm 0,11$ $\mathrm{g} / \mathrm{l}$ en los controles, pero en las cabras intoxicadas se registró un incremento progresivo que alcanzó valores medios de $0,46 \pm 0,11 \mathrm{~g} / 1$ al momento del sacrificio (Figura 1).

Similar comportamiento reveló la creatininemia, registrando valores de $1,35 \pm 0,64$ y $8,94 \pm 1,59 \mathrm{mg} / \mathrm{l} \mathrm{en}$ controles y tratados, respectivamente (Figura 2).

La densidad urinaria en las cabras del grupo experimental fue inicialmente de $\mathrm{P}_{\mathrm{e}} 1021 \pm 0.008$, pero hacia el final del período de intoxicación se elevó promediando $\mathrm{P}_{\mathrm{e}} 1024 \pm 13(\mathrm{p}<0.01)$, con rangos de $1010 \mathrm{a}$ 1037. En ambos grupos, el pH de la orina se mantuvo entre 8 y 9 sin variaciones durante la experiencia. No fue registrada la presencia de nitritos, glucosa, cuerpos cetónicos ni sangre. En cambio, al inicio de la intoxicación aparecieron vestigios de proteínas, que fueron incrementándose hasta alcanzar niveles que variaron entre 30 y $100 \mathrm{mg} / \mathrm{dl}$. En algunas cabras intoxicadas la bilirrubina, inicialmente negativa, registró trazas al final del ensayo, en tanto que el urobilinógeno permaneció normal en todos los casos.

En los sedimentos urinarios examinados previamente a la intoxicación se observó regular cantidad de células de transición, algunos cilindros granulosos y hialinos, así como escasa cantidad de eritrocitos y leucocitos (cinco y dos por campo microscópico respectivamente). A partir de la cuarta semana de iniciada la intoxicación, se registró un incremento progresivo de la cantidad de células de transición. 
Los órganos analizados manifestaron anormalidades de variada intensidad, siendo los hallazgos mas relevantes las lesiones histopatológicas del tejido pancreático de las cabras intoxicadas, en el cual se observaron numerosas vacuolas intracitoplasmáticas de pequeño tamaño en las células acinares de la porción exocrina. Algunas de las vacuolas presentaron bordes difusos y en algunos casos desplazamiento del núcleo (Figura 3).

En el tejido renal de los animales experimentales se verificó leve vacuolización citoplasmática y degeneración turbia de las células tubulares, cuyos núcleos conservaron su tamaño uniforme. A nivel intratubular se constató abundante contenido eosinofilico, presente en todos los tramos del sistema tubular.

El diámetro medio de los glomérulos de las cabras controles fue de $130,53 \pm 11,87 \mu \mathrm{m}$ y el de las cabras intoxicadas resultó de 146,03 $\pm 19,03 \mu \mathrm{m}$; la diferencia entre ambos resultó estadísticamente no significativa $(p>0,05)$. Sin embargo, el espacio de Bowman, casi

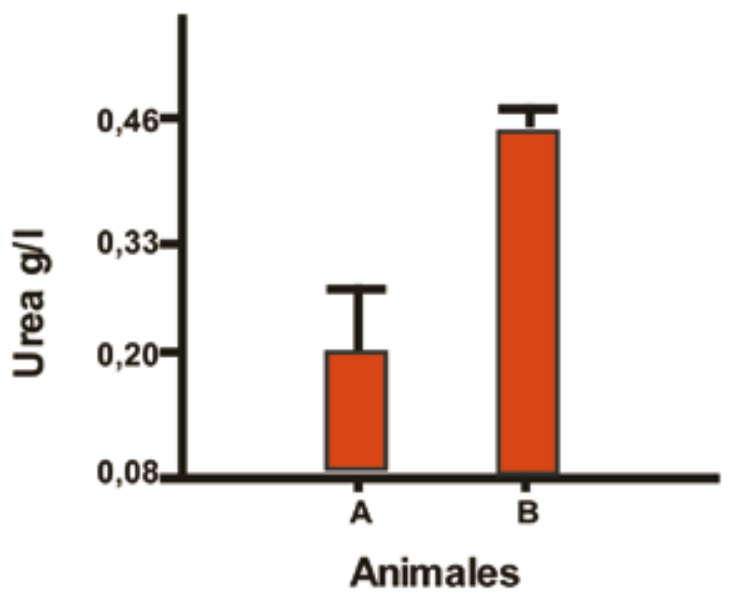

Figura 1. Valores séricos de urea en cabras controles (A) e intoxicadas (B), $\mathrm{X} \pm \mathrm{DE}(\mathrm{p}<0,0001)$.

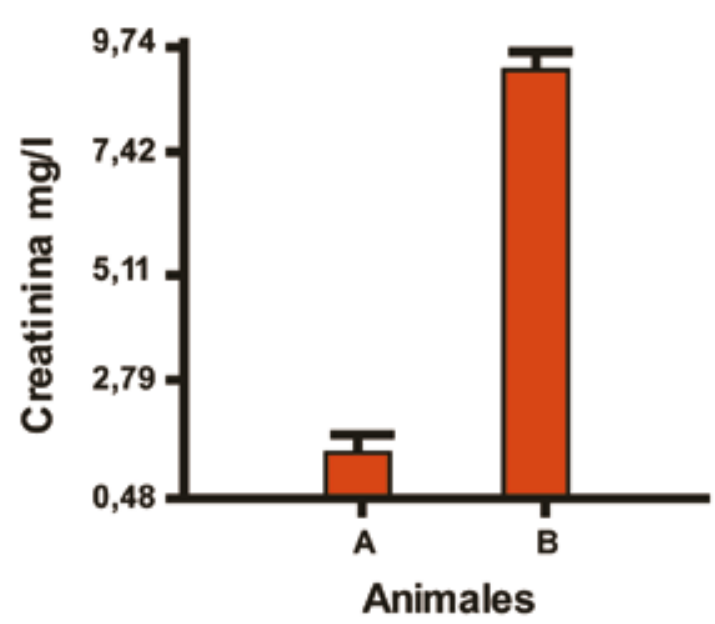

Figura 2. Valores séricos de creatinina en cabras controles (A) e intoxicadas (B), $\mathrm{X} \pm \mathrm{DE}(\mathrm{p}<0,0001)$. inexistente en las cabras controles (Figura 4), se ensanchó en las intoxicadas hasta una amplitud media de 31,63 $\mu \mathrm{m}$ (Figura 5). Ello indica que el aumento del diámetro del corpúsculo de Malpighi de las cabras tratadas se debió al ensanchamiento del espacio de Bowman.

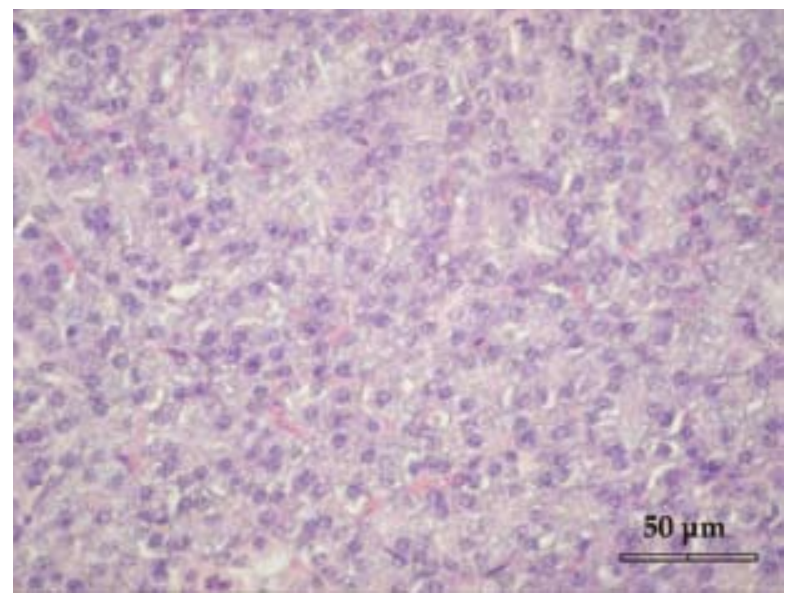

Figura 3. Páncreas de cabra intoxicada (HyE). Se observan abundantes vacuolas intracitoplasmaticas en células acinares exocrinas.

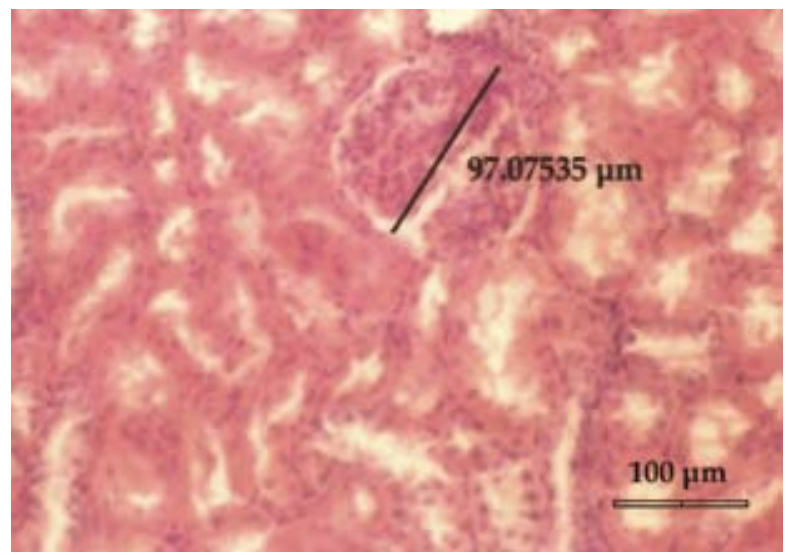

Figura 4. Riñón de cabra control (HyE). Se aprecia el diámetro de un glomérulo $(97 \mu \mathrm{m})$, donde el espacio de Bowman es casi inexistente. HyE.

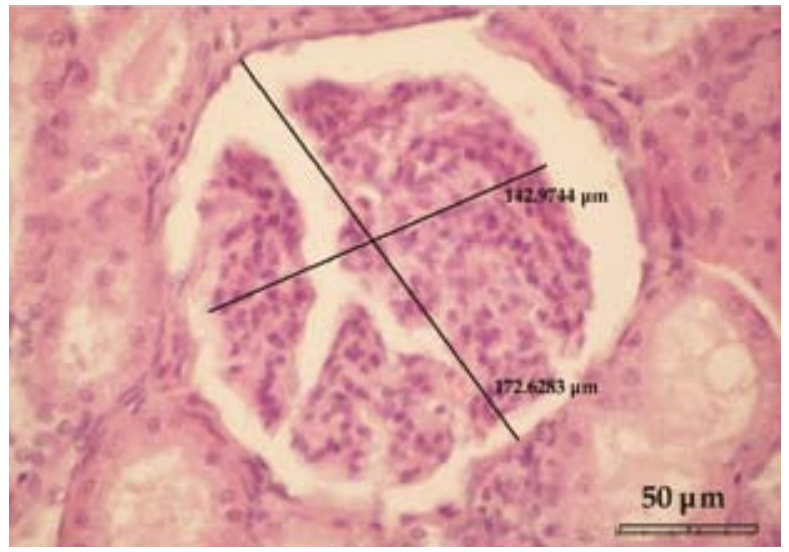

Figura 5. Riñón de cabra intoxicada (HyE). Los diámetros del corpúsculo de Malpighi $(172 \mu \mathrm{m})$ y del penacho glomerular $(142 \mu \mathrm{m})$ indican que el espacio de Bowman está aumentado (a unos $30 \mu \mathrm{m}$ ). También se aprecian vacuolas en las células tubulares. 


\section{DISCUSIÓN}

La vacuolización citoplasmatica en tejidos pancreático y renal de cabras intoxicadas con I. carnea es coincidente con lesiones similares halladas en caprinos sometidos a la ingestión de la planta, daños que son atribuidos al alcaloide swainsonina ${ }^{1}$. En experiencias anteriores la intoxicación de cabras con I. carnea recogida en primavera, alcanzó su máxima gravedad a los 30 días de iniciada la ingestión ${ }^{16,17}$, mucho antes del lapso registrado en el presente trabajo (53 días). Este hecho sugiere que la toxicidad de la planta varía según la época del año.

En otros estudios se emplearon dosis diarias menores $(5 \mathrm{~g} / \mathrm{kg}$ peso) a las del presente ensayo, administrando hojas de la misma y otras especies (I. sericophylla, I. riedelii) durante períodos comprendidos entre 22 y 105 días, sin registrarse grandes diferencias en los síntomas clínicos y lesiones histopatológicas entre las cabras asignadas a los distintos experimentos ${ }^{1,2}$.

Al inicio de la intoxicación los animales se resistieron a la ingestión de la planta, luego de una semana la aceptaron y a partir de allí la consumieron con gran avidez, seleccionando I. carnea entre otros verdeos disponibles. Esta preferencia o facilitación social por el consumo de la planta ya había sido descripta por otros autores en distintas variedades del género Ipomoea y en otras plantas que contenían swainsonina ${ }^{11}$.

Acorde a la tipificación convencional de las lesiones histopatológicas del páncreas exocrino, los daños aquí registrados deben ser considerados como intensos ${ }^{10}$. La vacuolización citoplasmática de las células glandulares podría haber afectado la síntesis de enzimas digestivas y explicar, en parte, la pérdida de masa muscular de los caprinos por impedimento de la normal digestión intestinal y consecuente alteración en la absorción de nutrientes necesarios para la síntesis proteica, todo ello sumado a la alteración del hígado, órgano que también es afectado por esta planta, pese a que en las cabras intoxicadas en el presente ensayo, la bilirrubinuria no fue acompañada por ictericia ${ }^{1,15}$.

En el tejido renal se confirmó la vacuolización citoplasmatica descripta por otros autores en esta intoxicación ${ }^{1,15}$ y además se hallaron lesiones no reportadas con anterioridad, como el ensanchamiento del espacio de Bowman y la presencia de contenido eosinofílico intratubular. Estos daños renales fueron concomitantes con la aparición de proteinuria y con el incremento de las concentraciones séricas de urea y creatinina, indicando alteraciones de filtración y secreción tubular. Tales disfunciones son compatibles con el bloqueo de la luz tubular y con la separación de las capas parietal y visceral de la cápsula de Bowman anteriormente descriptas.

La significativa disminución de la densidad urinaria de algunas cabras intoxicadas, hasta valores tan bajos como $\mathrm{P}_{\mathrm{e}} 1010$, sugiere pérdida de la capacidad de concentrar la orina por parte de los riñones. Otros animales, por el contrario, incrementaron la densidad urinaria a valores extremos de $\mathrm{P}_{\mathrm{e}}$ 1037, fenómeno que podría ser atribuido a la deshidratación, tal como fue demostrado en la intoxicación provocada por la planta recogida en primavera ${ }^{16}$.

En conclusión, surge que las cabras intoxicadas con I. carnea, además de las lesiones pancreáticas y renales descubiertas por otros autores, registran ensanchamiento del espacio de Bowman y presencia de contenido eosinofílico en la luz de los túbulos renales, hallazgos que explican los cambios urinarios y las elevaciones séricas de urea y creatinina, circunstancias a ser tenidas en cuenta al momento de efectuar el estudio toxicológico.

\section{REFERENCIAS}

1. Armién AG, Tokarnia CH, Vargas Peixoto P, Frese K. 2007. Spontaneous and experimental glycoprotein storage disease of goats induced by Ipomoea carnea subsp fistulosa (Convolvulaceae). Vet Pathol 44: 170-184.

2. Barbosa RC, Riet Correa F, Medeiros RM, Lima EF, Barros SS, Gimeno EJ, Molyneux RJ, Gardner DR. 2006. Intoxication by Ipomoea sericophylla and Ipomoea riedelii in goats in the state of Paraíba, Brazil. Toxicon 47: 371-379.

3. Cholich EJ, Gimeno PG, Teibler NL, Jorge OC, Acosta O. 2009. The guinea pig as an animal model for Ipomoea carnea induced a-mannosidosis. Toxicon 54: 276-282.

4. Daló N, Moussatché H. 1978. Acción tóxica de las plantas del género Ipomoea. Revista de la Universidad Centro Occidental (Venezuela) 6: 25-39.

5. De Balogh K, Dimande AP, Van Der Lugt JJ, Molyneux RJ, Naudé TW, Welman WG. 1999. A lysosomal storage disease induced by Ipomoea carnea in goats in Mozambique. J Vet Diag Inv 11: 266-273.

6. Gardner D, Lee S, Molyneux R, Edgar J. 2003. Preparative isolation of swansonine from locoweed: extraction y purification procedures. Phyt Anal 14: 259-266.

7. Haraguchi M, Gorniak SL, Ikeda K, Minami Y, Kato A, Watson AA, Nash RJ, Molyneux RJ, Asano N. 2003. Alkaloidal components in the poisonous plants, Ipomoea carnea (Convolvulacea). J Agric Food Chem 51: 4995 5000 .

8. Hoehne FC. 1939. Plantas e substancias tóxicas e medicinais. Ed. Graphics, São Paulo, 166 p.

9. Hueza IM, Dagli ML, Gorniak SL, Paulino CA. 2003. Toxic effect of prenatal Ipomoea carnea administration to rats. Vet Hum Toxicol 45: 298-302.

10. Jeraldo TL, Coutu JA, Verdier PA, McMillan PN, Adelson JW. 1996. Fundamental cellular heterogeneity of the exocrine pancreas. J Histochem Cytochem 44: 215-220.

11. Jolly RD, Walkley SU. 1997. Lysosomal storage disease of animals: an essay in comparative pathology. Vet Pathol 34 : 527-548.

12. Molyneux RJ, Pan YT, Goldmann A. 1993. Calystegins, a novel class of alkaloid glycosidase inhibitors. Arch Bioch Biop 304: 81-88.

13. Molyneux RJ, Mckenzie RA, O'Sullivan BM, Elbein AD. 1995. Identification of the glycosidase inhibitors swainso- 
nine and calystegine B2 in weir vine (Ipomoea sp.) and correlation with toxicity. J Nat Prod 58: 878-886.

14. Ralphs MH, Panter KE, James LF. 1990. Feed preferences and habituation of sheep poisoned by locoweed. $J$ Anim Sci 68: 1354-1362.

15. Riet-Correa E, Mendez M. 2000. Plantas tóxicas e micotoxicoses, Ed. Gráfica Universitária, Pelotas (Brasil), p. 36-37.

16. Ríos E, Belmonte C, Rodríguez C, Ortiz l, Ciotti E, Bogado F, Acosta de Pérez O. 2005. Intoxicación con Ipomoea fistulosa (aguapié o mandiyurá) en cabras. Efectos sobre el hemograma e ionograma. Rev Vet 16: 21-24.
18. Ríos E, Cholich L, Silva J, Acosta de Pérez O. 2008. Histopathological lesions in central nervous system of goats poisoned by Ipomoea carnea. Rev Vet 19: 130-134.

19. Stegelmeier BL, Molyneux RJ, Elbein AD, James LF. 1995. The lesions of locoweed (Astragalus mollissimus), swainsonine, and castanospermine in rats. Vet Pathol 32: 289-298.

20. Tokarnia CH, Dobereiner J, Peixoto PV. 2002. Poisonous plants affecting livestock in Brazil. Toxicon 40: $1635-1660$. 